\title{
EP Communications: The Next Revolutionary Step in Global Communications
}

\author{
Paul W. Phister, Jr. \\ Steven L. Drager \\ Igor G. Plonisch \\ Air Force Research Laboratory / Information Directorate \\ 26 Electronic Parkway, Rome NY 13441-4514 \\ 315-330-3315 \\ paul.phister@rl.af.mil \\ steve.drager@el.af.mil \\ igor.plonisch(arl.af.mil
}

\begin{abstract}
Throughout time, humankind has always moved towards more optimal methods of communications. Since the late $1800 \mathrm{~s}$, the age of Radio Frequency (RF) has dominated the medium in which we have communicated. Over the past 50-plus years, the RF spectral band has become increasingly overcrowded. The time is right for investigating the next step in the evolution of global communications. One possible evolutionary step in communications is Entangled-Pair (EP) communications. This paper discusses the significant advantages an EP communications system would have over today's RF and Optical communications architectures. A theoretical background regarding the entangled-pair phenomena, along with the current state-of-the-art in EP communications, is presented. This paper makes a leap ahead in time (i.e., assumes an operational EP communications system) to compare and contrast what a fully functional EP communications system could provide. A summary of the required EP technologies and their current Technology Readiness Levels (TRL) is provided, along with a Science and Technology roadmap.
\end{abstract}

\section{TABLE OF CONTENTS}

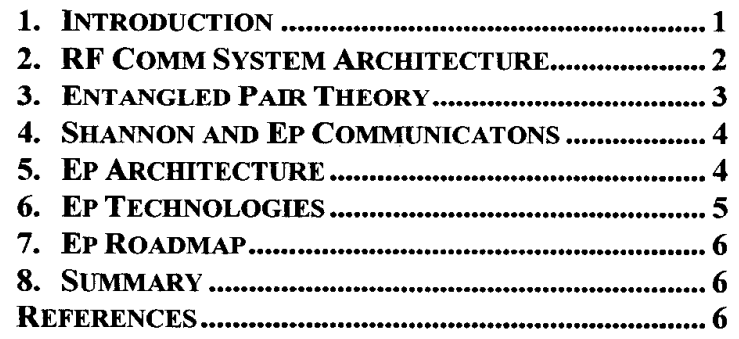

\section{INTRODUCTION}

Throughout time, humankind has always moved towards more optimal methods of communications. Since the late 1800 s, the age of Radio Frequency (RF) has dominated the medium in which we have communicated. Consequently, over the past 50-plus years, the RF spectral band has become so crowded that we have had to continually invent new methods to cram more information into the "communications pipes," or make information travel down the "communications pipe" faster. However, this cannot continue. This paper explores a concept that could take today's communications methodologies to the next level, namely, quantum communications using entangled-pairs (EP). The first part of the paper lays the theoretical foundation of "EP Communications" and the current stateof-the-art. The latter part of the paper leaps ahead 30-plus years (to 2035) and assumes there is a fully functional EP communications capability. Given the EP communications capability, an example is used to illustrate its utility, and how it would virtually revolutionize satellite communications by eliminating the requirement for relay satellites. RF or optical spectral bands would not be used: quantum communications using entangled-pairs is virtually instantaneous with unlimited bandwidth.

\section{RF COMM SYSTEM ARCHITECTURE}

The primary reason for space communications today is to solve the beyond-line-of-sight (BLOS) limitations in pointto-point communications. There are numerous ways to communicate with a space vehicle using the existing space architecture; namely:

a) directly, while the space vehicle is within line-of-sight (LOS); or

b) via a relay node, as in the Air Force Satellite Control Network to "hop" to the space vehicle; or,

c) via a relay satellite.

These methods are depicted in Figure 1.

This method of communications has grown exponentially over the past ten years, and it appears the only way to solve the problem is to put additional communications systems in space. This has caused the RF spectral band to be extremely crowded, as shown in Figure 2. The spectral bands are so crowded today, that in some instances, the band is "doublebooked" and techniques have to be employed to ensure the two "users" do not interfere with each other. 


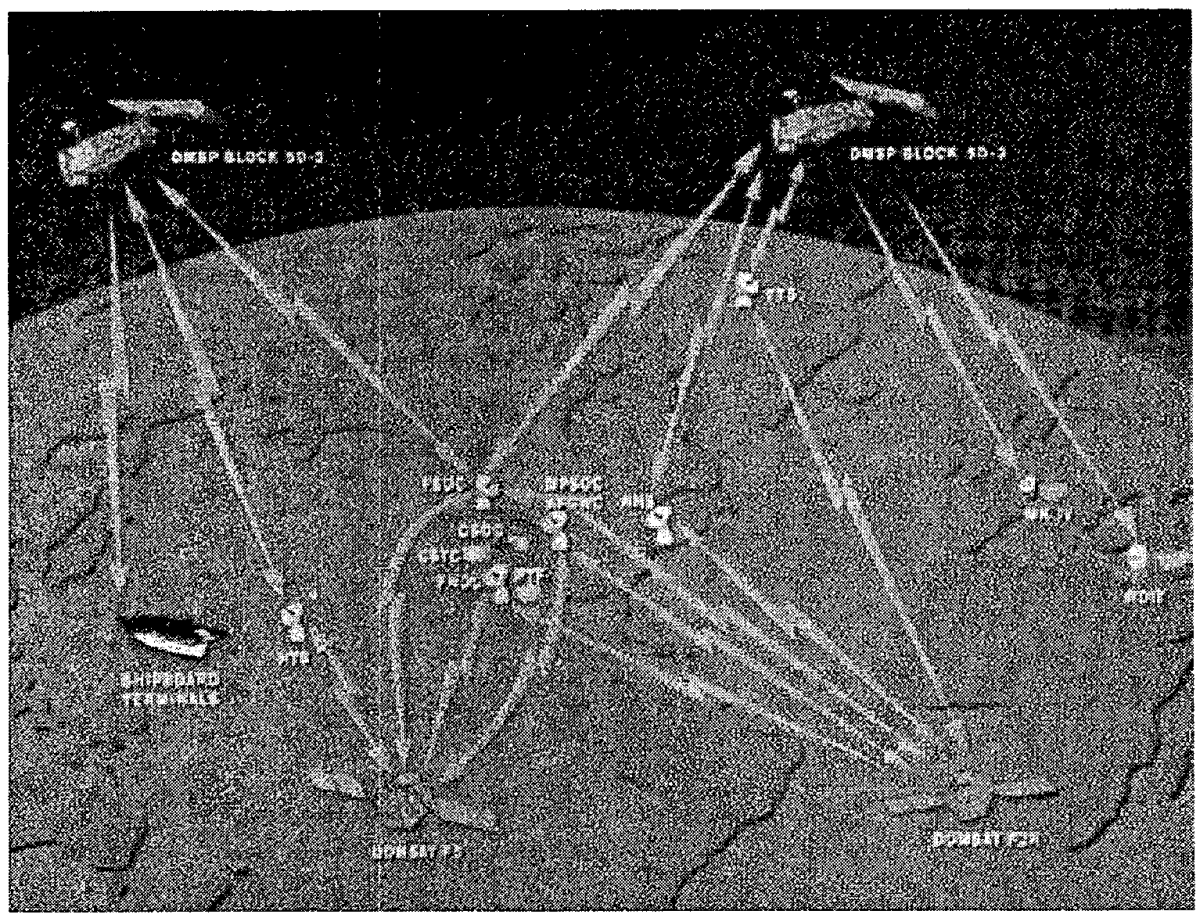

Fig. 1. Satellite Communications Architecture

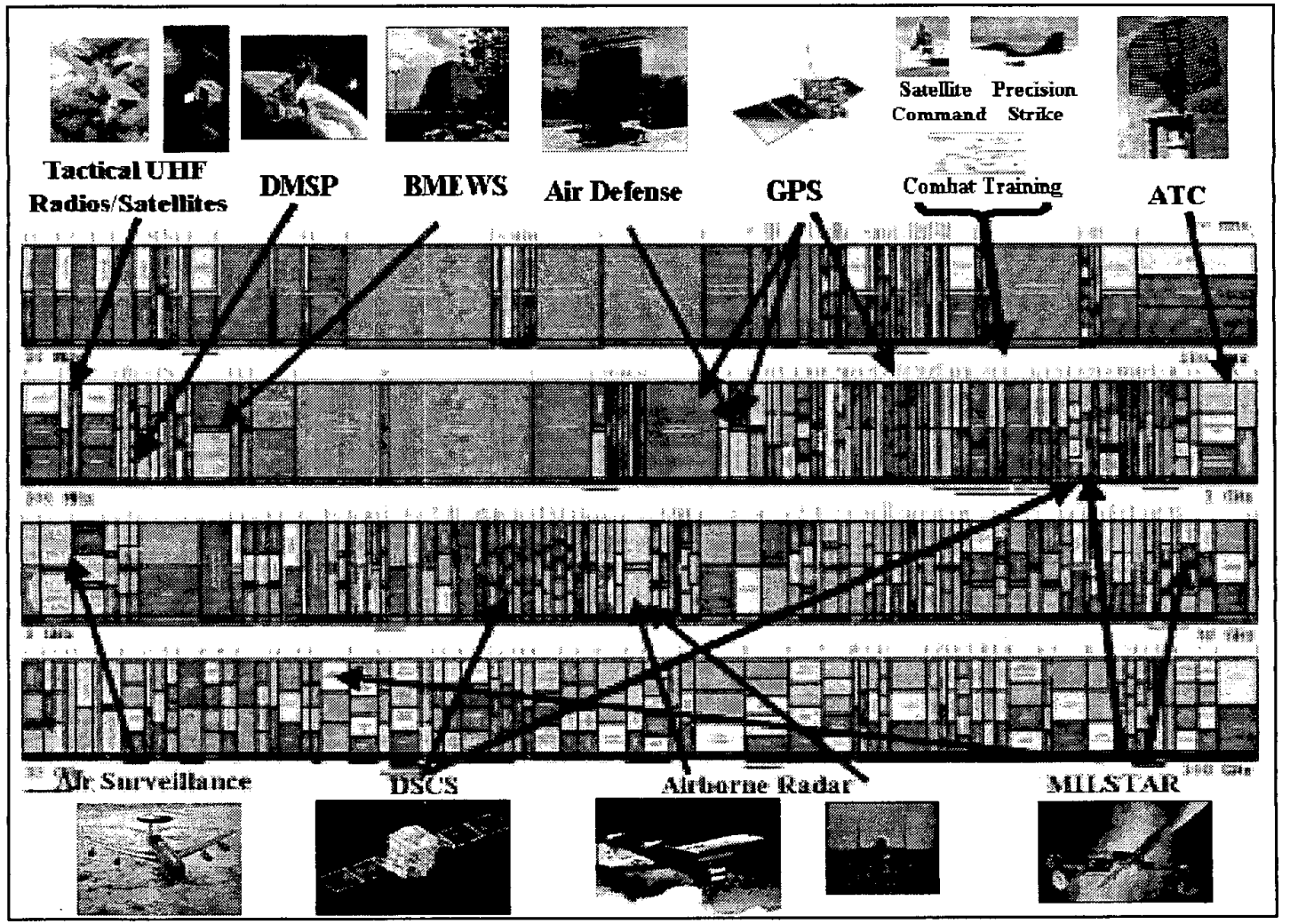

Fig. 2. Current RF Spectral Band Usage 
The current space architecture can be thought of as subdivided into three main areas: a space segment, a ground segment, and an air segment as shown in Figure 3. The links that enable communications are ground-to-space, air-tospace and space-to-space. Given the frequency allocation, it takes both bandwidth and throughput rate to successfully transmit data from the space vehicle to the mission ground station (via a variety of paths as outlined in Figure 3 ). This can be a time consuming process. For example, to completely transmit a 3500 band $(1024 \times 1024)$ hypercube, it would take approximately 10 -mins at a $50 \mathrm{Mbps}$ rate [3500 bands $\times(1.05 \mathrm{Mpixels} / \mathrm{band}) \times(8 \mathrm{bits} / \mathrm{pixel}) \times$ $\left.\left(1 / 50 \times 10^{6} \mathrm{bits} / \mathrm{sec}\right) \times(1 \mathrm{~min} / 60 \mathrm{sec})\right]$. Considering a low earth orbit vehicle (approximately 90 -mins per revolution), then allowing for a 10 -min downlink time per hypercube, and having a 45-min window, hyperspectral images are limited to just four per operational window. This means that unless there is sufficient memory on-board the vehicle, the mission planning system would have to limit the images to just four per orbit. This is not an effective use of a highly complex, versatile, and expensive system.

As noted, Figure 3 illustrates a generic space architecture. Given the state-of-the-art in communications, this architecture is limited to the method (and corresponding speed) of communications (RF, Optical, Laser). These three segments must work together to continuously provide a link to the space vehicle and a corresponding link to the end user for information to be transferred.

\section{Entangled Pair Theory}

The understanding of quantum mechanics phenomena is necessary to be able to view the future of communications. These are entangled quantum states and quantum teleportation.

Entangled quantum states are a very important cornerstone of quantum computing and communication. They lay the foundation for such important concepts as quantum error correction and quantum teleportation. As discussed by Smolin, a quantum state is a complete description of a system at one moment in time, according to the quantum theory [1]. Entanglement is a characteristic trait of quantum mechanics. It forces one to depart from classical lines of thinking. Consider the two-qubit state:

$$
|\psi\rangle=\frac{|00\rangle+|11\rangle}{\sqrt{2}}
$$

This state has the remarkable property that there is no single qubit states $|a\rangle$ and $|b\rangle$ such that $|\psi\rangle=|a\rangle|b\rangle$.

A composite system having this property - that of not being written as a product of the states of its component systems - is entangled.

Entanglement is a characteristic trait of quantum mechanics that occurs when a pair of particles is simultaneously created. What is unique is that there does not have to be a communications channel linking the sender to the recipient for communication to occur.

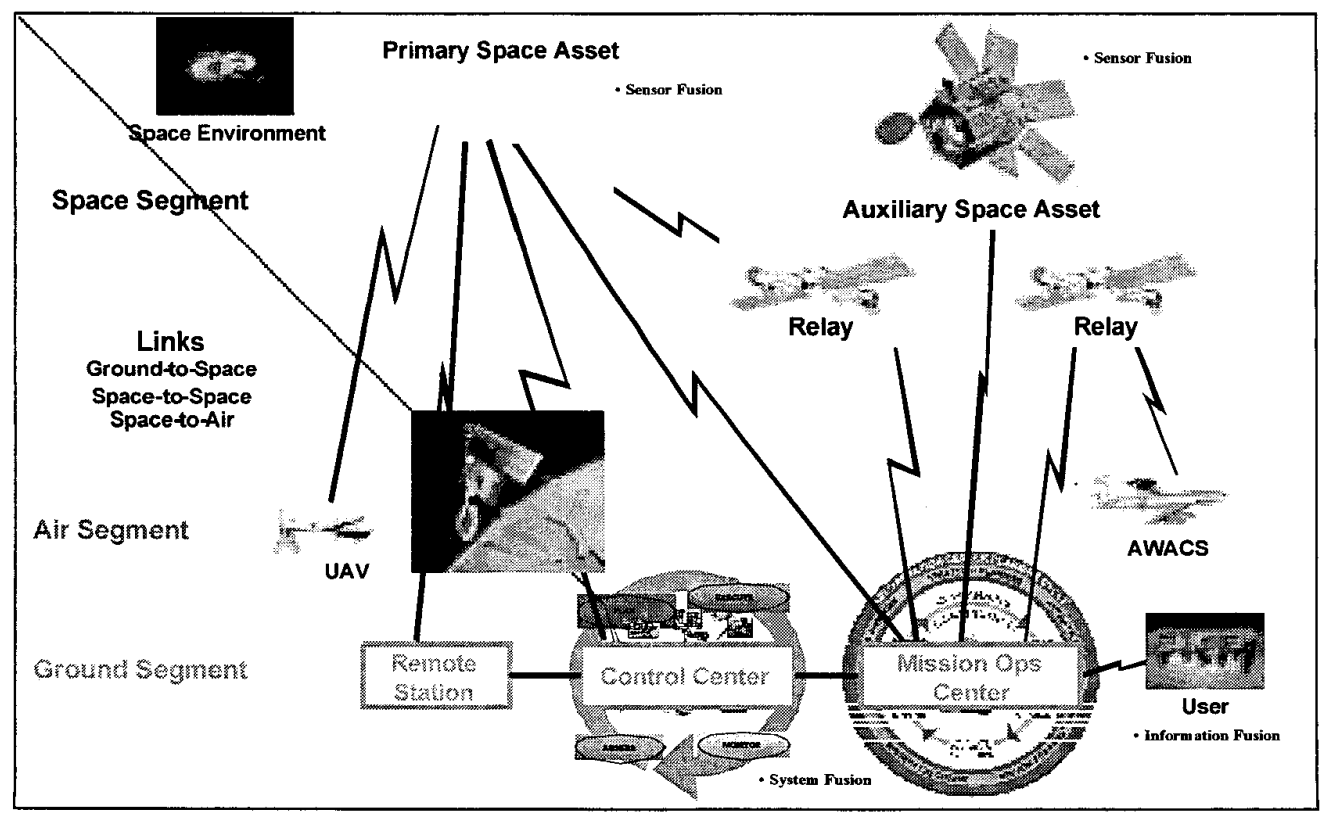

Fig. 3. Generic Space Architecture 
Quantum teleportation involves transferring a state from one particle to another without that state having to move through the intervening space. Key to this concept is that the particle does not cross the intervening space, but rather is scanned for sufficient information, transmitted and an exact replica is recreated at the destination. Quantum teleportation is a concept that requires thinking beyond classical lines of thought. Quantum teleportation affects the faithful transfer of an unknown quantum state through a potentially hostile environment by using classical bits. [2] [3] How might a communications system built on these concepts work? [3] To follow conventional format, Alice and Bob are best friends. They have now moved very far apart, but when they were together they generated a special qubit pair, an Einstein, Podolsky, Rosen (EPR) pair. Now when Alice and Bob are separated, each of them took one of the qubits with them. Due to entanglement, even though there is separation, the influence of one particle over the other still persists (This influence exists between the two qubits no matter what the distance and no matter what medium is separating the two particles). Alice mixes her half of the EPR pair with the qubit she wishes to send to Bob, measures the result and sends the qubit and classical measurement results to Bob. Bob is then able to reconstruct the qubit using his half of the EPR pair. This is quantum teleportation and a method of information transfer.

A few points require clarification. First, quantum teleportation does not transmit the information faster than light, as there is a classical component involved. This follows from Einstein's basic principle that "nothing can travel faster than the speed of light." [4] Second, classically describing the qubit would take an infinite amount of information, as the qubit is continuous. Finally, this type of information transmission has some inherent security associated with it. For example, without one of the EPR pair halves, a third person, Eve, would not be able to reconstruct the teleported qubit between Alice and Bob.

\section{ShaNNON AND EP COMMUNICATIONS}

Communications is the process whereby information is transferred from one point in space and time, called the source, to another point, the destination or user [5].

Figure 4 is a simplistic view of Shannon's generic communications channel [6]. As defined, this channel is the electrical channel between transmitter and receiver, bridging the distance from source to destination. Today this is a pair of wires, a coaxial cable, a radio wave, or even a laser beam. [5] It is well understood that the fundamental limitation of information transmission by electrical means are bandwidth and noise [7]. An EP communications system does not have the traditional noise insertion component caused by channel losses (e.g., atmospherics) that a normal RF or optical communications system experiences. This is due to the fact that the photons are "entangled" and can be separated an infinite distance as if they were right next to each other. An EP communications link is shown in Figure 5. Naturally, even communications via an entangled pair will have some form of noise or distortion in the system. However, what form this will take and its impact on a communications system are unknown at this time. One needs to point out that by utilizing the entangled-pair as a communications medium, there is no need for the traditional transmitter and receiver antennas (e.g. bandwidth). Since the two entangledpairs communicate directly at the quantum level, instead of a $66-\mathrm{ft}$ transmitter and $30-\mathrm{ft}$ receiver antenna for space communications, the transmitter and receiver can be thought of as part of the electronics and are the size of two photons. [5] [8] [9]

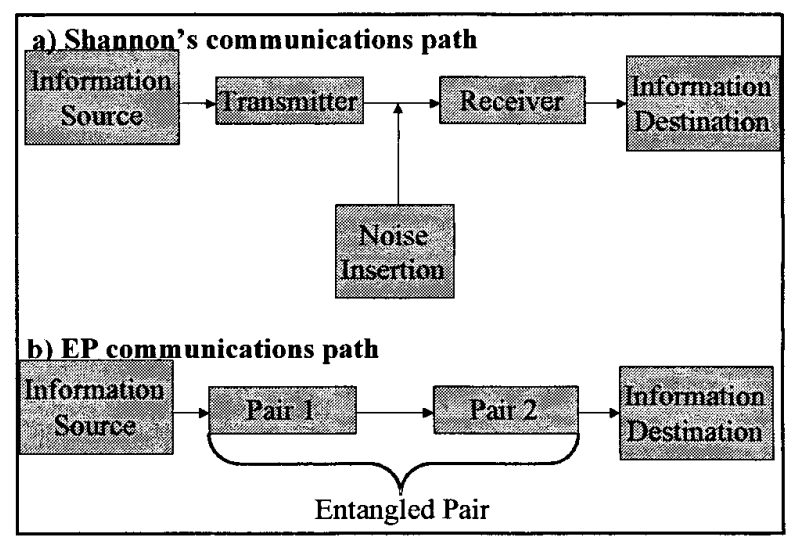

Fig. 4. Generic Communications Channel

The classical communications metrics used today have little to no meaning when discussing EP communications. For example, there is no bandwidth. It is thought that Shannon's symbol transfer rate (symbols per second) is a better metric to use when describing the transfer rate of information from Source to Destination.

\section{EP ARCHTECTURE}

Let us assume that we have a working device that allows us to fully utilize an EP communications architecture (as shown in Figure 5). Since the entangled pair can be separated by an infinite distance, there is no line-of-sight (LOS) constraint. Consequently, a mission ground station (or user) will have practically unlimited visibility to the space vehicle throughout its orbit. This is analogous to having geostationary-earth-orbit (GEO) characteristics at low-earth-orbit (LEO). In other words, this negates the requirement for the use of either a relay communications satellite or the Air Force Satellite Control Network to communicate with the space vehicle. Conceptually, this is shown in Figure 5 where the only "links" that are required are essentially point-to-point to/from the space vehicle. 


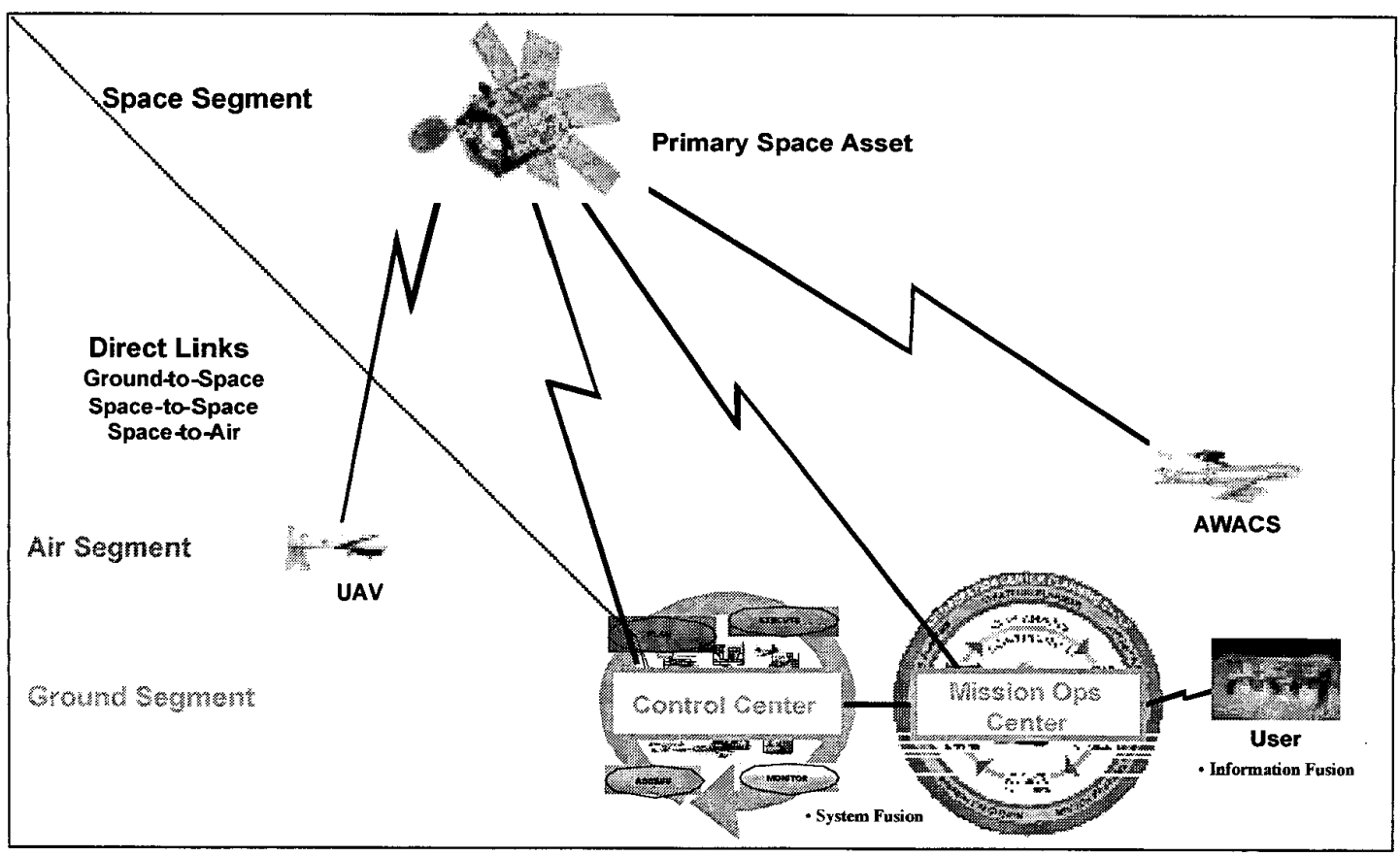

Fig. 5. EP Communication Paths

Using the same example of a 3500-band hypercube, the complete hypercube would be transmitted to the mission ground station virtually instantaneously. Therefore, given a 45-minute operations window and allowing for a 15-sec imaging time, this means that 180 hypercubes could be planned instead of the earlier example of four using conventional communications technology. This represents several orders of magnitude improvement in communications capabilities. This symbol transfer rate allows 3500 band hyperspectral imagery to be downlinked directly from a space vehicle to the mission ground station (anywhere in the orbit without the need for a relay communications satellite) in micro or nano seconds!

In addition, if the mission ground station had a quantum computer, the hypercube could be processed in one clockcycle! Having a quantum computer aboard the space vehicle would mean that an entire mission ground station would be on-board the vehicle and would perform the entire TaskingCollection-Processing-Exploitation-Dissemination (TCPED) process in nanoseconds!

As mentioned earlier, since EP communications is really a quantum level method of communicating, its communications metrics cannot be expressed in the traditional manner (e.g., bandwidth, bps). It is thought that Shannon's symbol transfer rate is a more appropriate metric.

\section{EP TECHNOLOGIES}

EP communications require research investment over the next 30-35 years. The technologies requiring investment are:
a) Quantum "entangled-pair"
b) Quantum computing
c) Quantum communications.

As mentioned above, the transmitter and receiver would be a set of entangled pairs. If we can get them to stay entangled (forever) then the transmitter and receiver can be thought of as an infinitely long linear predictive coder. This provides for virtually instantaneous communications from Alice to Bob and Bob to Alice. [3]

As a measure of technology readiness, the Air Force Research Laboratory utilizes NASA's Technology Readiness Level (TRL) curves. The NASA TRL scale is based on nine levels. Figure 6 illustrates the first six levels. Historically, the Air Force Research Laboratory works on Levels 1-6 (Science and Technology) and then transitions the technology to either Product Centers or Industry.

Currently, the work being performed in quantum information sciences is at the TRL- 1 and TRL-2 levels, at best. 


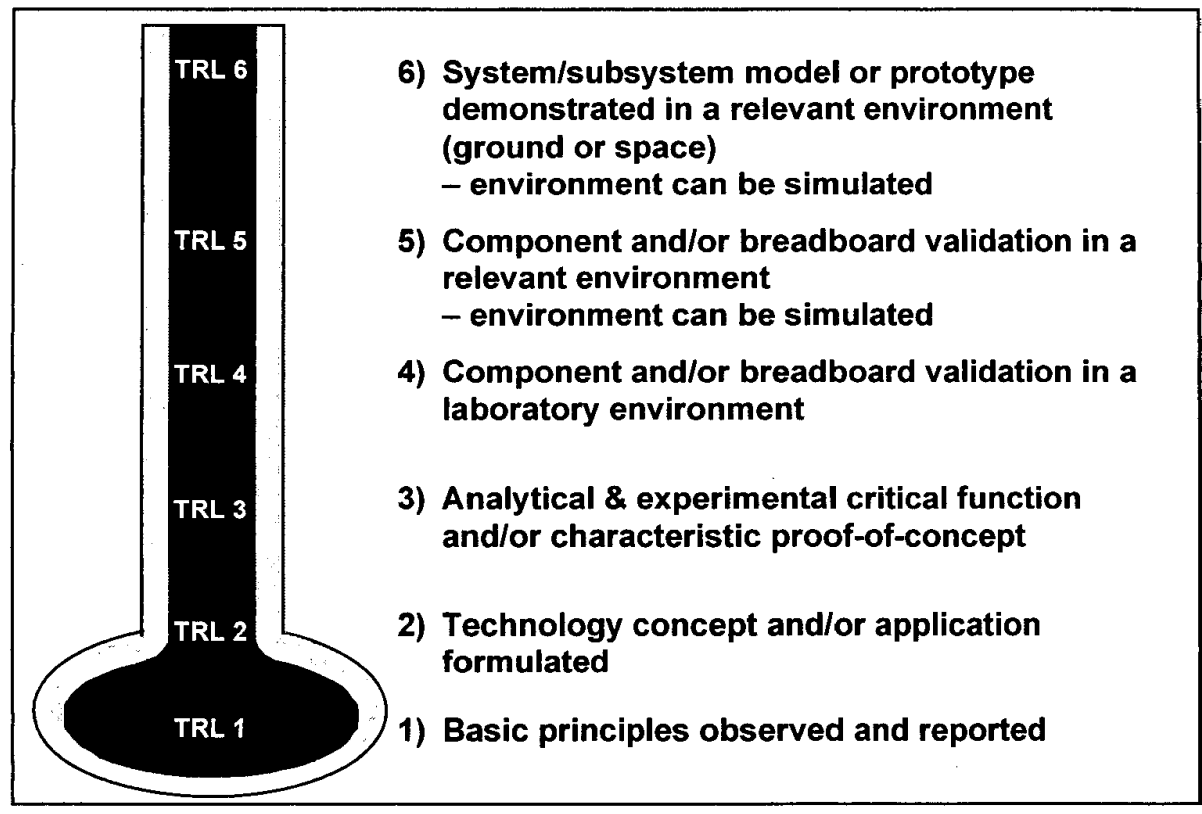

Fig. 6. NASA's Technology Readiness Levels 1-6.

\section{EP ROADMAP}

The basic problem to be solved is the ability to have a permanently entangled pair. Currently, we can create an entangled pair, but it is far from permanent. The creation of a permanently entangled pair is definitely the "very long, long, long pole in the tent." The long-term basic research roadmap in quantum computing and quantum communications needs to be accomplished. It is envisioned that this will take approximately 25-30 years of basic research in the Laboratory in conjunction with Industry and Academia to be fully successful.

\section{SUMMARY}

EP communications can be thought of as taking that next step toward a higher level of communications capability. This method can have significant payoffs in satellite communications as it could virtually negate the requirement for relay satellites.

The Air Force Research Laboratory's Information Directorate, located in Rome NY is performing basic research in quantum information sciences with both Industry and Academia. Currently, this is a modest research budget; however, the payoffs will be staggering.

\section{REFERENCES}

[1] Lee Smolin, Three Roads to Quantum Gravity, Basic Books, 2001, pages 34, 218.

[2] Timothy P. Spiller, "Quantum Information Processing: Cryptography, Computation, and Teleportation," Proceedings of the IEEE, Vol 84., No 12., Dec 1996, pages 1719-1746.
[3] Anton Zeilinger, "Quantum Teleportation", Scientific American April 2000, pages 50-59.

[4] Richard J. Gott, Time Travel in Einstein's Universe, Houghton Mifflin Company, 2001, pages 53-55.

[5] A. Bruce Carlson, Communications Systems: An Introduction to Signals and Noise in Electrical Communications, McGraw-Hill, 1975, pages 3-8.

[6] Michael A. Nielsen and Isaac L. Chuang, "Quantum Computation and Quantum Information," Cambridge University Press, 2000.

[7] Yorick Hardy and Willi-Hans Steeb Birkhauser, "Classical and Quantum Computing," Verlag, 2001.

[8] Colin P. Williams and Scott H. Clearwater Springer, "Explorations in Quantum Computing," Verlag, 1998.

[9] Robert G. Gallager, Information Theory and Reliable Communications, John Wiley and Sons, 1968, Chapter 1.

\section{BIOGRAPHIES}

Paul W. Phister, Jr., Ph.D., P.E., is the Air and Space Strategic Planner at the Air Force Research Laboratory's Information Directorate headquartered in Rome, New York. Dr. Phister received his Ph.D. in Engineering from California Coast University and is a licensed Professional Software Engineer from the State of Texas.

Steven Drager is the Technical Advisor for the Advanced Computing Architecture Branch at the Air Force Research Laboratory's Information Directorate headquartered in Rome, New York.

Igor Plonisch is the Chief of the Strategic Planning and Business Operations Division at the Air Force Research Laboratory's Information Directorate headquartered in Rome, New York. 